\title{
Role of computed tomography in diagnosis of bronchiectasis
}

\author{
J C COOKE, D C CURRIE, A D MORGAN, I H KERR, D DELANY, \\ B STRICKLAND, P J COLE
}

From the Department of Radiology, Brompton Hospital, and Host Defence Unit, Department of Thoracic Medicine, Cardiothoracic Institute, London

ABSTRACT Bronchograms and computed tomograms were performed in 27 patients who presented consecutively for bronchography with chronic sputum production. The films were reported separately by three consultant radiologists, who had been asked to give a diagnostic interpretation? of the films for each bronchopulmonary segment. The reporting of bronchiectasis on computed tomograms was compared with that on bronchograms. The sensitivity and specificity of computec. tomography at segmental level compared with bronchography was $66 \%$ and $92 \%$, respectively. W远 conclude that computed tomography alone is not yet suitable for accurate characterisation and localisation of disease in patients in whom surgery is contemplated. Computed tomography may be useful in patients in whom bronchography is contraindicated and for monitoring progression of disease after initial combined computed tomography and bronchography.

Bronchography has been used for many years as a definitive investigation to identify the presence, severity, and extent of bronchial abnormalities, particularly bronchiectasis, but it is an invasive investigation and unpleasant for the patient. Despite refinements in technique and contrast medium, complications may occur, which include allergic and foreign body reaction to the contrast medium, pulmonary infection, and a temporary reduction in pulmonary function with impaired ventilation and diffusion. ${ }^{1}$

Bronchiectasis, as the term is used in this study, is permanent, irreversible dilatation of the bronchial tree, and its features as seen by the newer technique of computed tomography (CT) have been described by Naidich et al. ${ }^{2}$ Mootoosamy et al recently concluded that CT was useful in assessing bronchiectasis, ${ }^{3}$ but Muller $e t a l$, on the other hand, found it unreliable in diagnosing cylindrical and varicose bronchiectasis. ${ }^{4}$

In view of the apparent conflict in current reports, we conducted a controlled study to assess the sensitivity and specificity of CT compared with bronchography in the diagnosis of bronchiectasis.

Address for reprint requests: Dr J Cooke, Department of Radiology, Royal Marsden Hospital, London SW3 6JJ.

Accepted 1 October 1986

\section{Patients and methods}

PATIENTS

Twenty seven patients who presented consecutively for bronchography with symptoms of cough and sputum were studied. Of these, 26 expectorated sputum daily (median duration eight years) and the् sputum in 24 was purulent; one expectorated sputumi intermittently. All but one of the patients were exam? ined more than three months after an exacerbation? the exception having a repeat CT scan three monthइ later; only the second scan was included in the analysis.

\section{IN VESTIGATIONS}

CT preceded bronchography in 24 patients; in the other three, CT followed bronchography by four to six weeks. The CT scans were performed on an Elscint 2002 scanner with a five second scanning time and 51 z $\times 512$ matrix. All patients were scanned supine, using a standard protocol, in full suspended inspiration from lung apex to diaphragm in $10 \mathrm{~mm}$ increment? with a $10 \mathrm{~mm}$ slice width. The optimum windowo width for visualising the bronchi was found to be 1400 to 1600 Hounsfield units (HU) with a centre of $-600 \mathrm{HU}$.

The CT scans were initially read by two pulmonar. radiologists, who were asked to provide a genera diagnostic interpretation of the scans for each seg 
ment, and to report on the presence or absence of five features for each segment-namely, proximal bronchial dilatation, distal bronchial dilatation, proximal bronchial wall thickening, distal bronchial wall thickening, and parenchymal shadowing.

Bronchography was performed in all 27 patients and each investigation preceded by a plain chest radiograph. The contrast was introduced into the lungs through a fibreoptic bronchoscope in 15 patients and a nasal catheter in 12 . Twenty three examinations were bilateral and four unilateral. The contrast agent used was an aqueous suspension of propyliodone (Dionosil Aqueous, Glaxo).

The bronchograms were assessed by two pulmonary radiologists for the presence or absence of proximal bronchial dilatation, distal bronchial dilatation, non-tapering of the bronchi, lack of filling of bronchial side branches, and presence of excess mucus (luminal filling defects). In addition, a diagnostic interpretation was reported for each segment (fig 1).

The radiologists were aware only that all patients had chronic sputum production. The patients' identity was concealed when the bronchograms and CT scans were reported. The two investigations were reported separately and in random order by both radiologists independently. A third radiologist assessed the bronchograms and CT scans in a similar manner but only recorded a diagnostic interpretation.

For each patient the plain chest radiograph was assessed for the presence of linear markings, "bronchial wall thickening", patchy or confluent pulmonary shadowing, pleural thickening, evidence of lung collapse or volume loss, and circular markings as described by Güdbjerg. ${ }^{5}$ In addition, the radiologists were asked to assess the presence or absence of bronchiectasis.

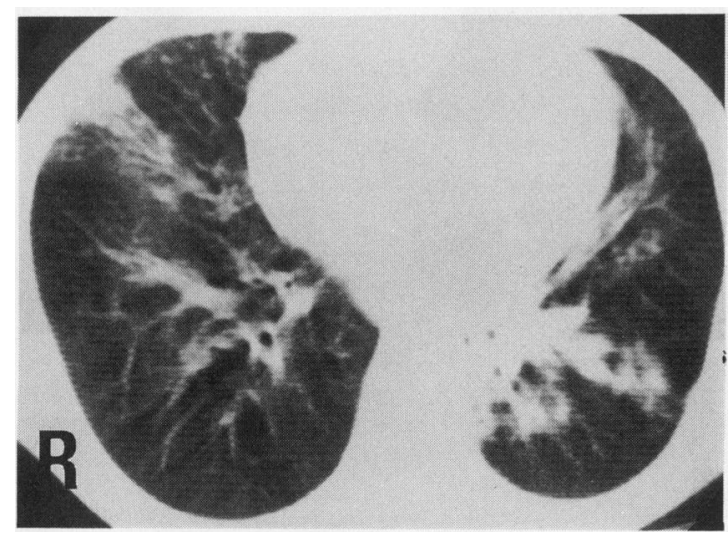

Fig 1 Computed tomogram, showing thick walled dilated bronchi, "tram lines", and peribronchial shadowing at both bases.
ANALYSIS

The diagnostic interpretations of all three radiologists were considered in the comparison of CT scans and bronchograms for each of the 448 segments. Bronchiectasis was defined as being present if it was reported by either two or three of the radiologists. Conversely, if only one or none of the radiologists reported bronchiectasis the segment was defined as non-bronchiectatic.

Bronchography was regarded as the "gold standard" against which CT was judged as it is the accepted method for diagnosing bronchiectasis. The sensitivity, false negative rate, specificity, and false positive rate of $\mathrm{CT}$ for the diagnosis of bronchiectasis that had been confirmed bronchographically were calculated at segmental, lobar, and lung level. Sensitivity (\%) was defined as:

$$
\frac{\text { True positive }}{\text { True positive }+ \text { false negative }} \times 100
$$

and was calculated at segmental level as:

$$
\begin{gathered}
\text { No of segments bronchiectactic } \\
\text { on both CT and bronchography } \\
\text { otal No of segments bronchiectactic } \\
\text { on bronchogram }
\end{gathered}
$$

The false negative $(\%)$ rate was $(100$ - sensitivity). Specificity (\%) was defined as:

$$
\frac{\text { True negative }}{\text { True negative and false positive }} \times 100
$$

and was calculated at segmental level as:

$$
\begin{gathered}
\text { No of segments non-bronchiectatic } \\
\text { on both CT and bronchography } \\
\begin{array}{c}
\text { Total No of segments non-bronchiectatic } \\
\text { on bronchography }
\end{array}
\end{gathered}
$$

and the false positive (\%) rate was (100 - specificity).

Similar calculations were used to derive these indices for CT at lobar and lung level. The detailed bronchographic interpretation of the abnormalities seen in the false positive segments on CT were recorded by the first two radiologists only. The correlations between CT and bronchography for the presence or absence of proximal dilatation and distal dilation were determined.

\section{Results}

Table 1 shows the number of segments reported as positive for each of the five features on CT. A total of 92 segments were reported as bronchiectatic by two or three radiologists. Table 2 shows the correlation between proximal and distal bronchial dilatation as reported on both CT and bronchography. The percentage of segments reported as positive on CT for proximal dilatation and distal dilatation/non-tapering was only $56 \%$ and $52 \%$ of 
Table 1 Diagnosis of bronchiectasis and abnormal features recorded in 448 segments on computed tomography. Values are No (\%)

\begin{tabular}{lccc}
\hline & $\begin{array}{l}\text { Reported by two } \\
\text { radiologists }\end{array}$ & $\begin{array}{l}\text { Reported by only one } \\
\text { radiologist }\end{array}$ & $\begin{array}{l}\text { All radiologists } \\
\text { reported absence }\end{array}$ \\
\hline $\begin{array}{l}\text { Diagnostic interpretation of bronchiectasis } \\
\text { Abnormal features: }\end{array}$ & $92 *(20)$ & $35(8)$ & $321(72)$ \\
$\quad$ Proximal wall thickening & $125(28)$ & $133(30)$ & $190(42)$ \\
Distal wall thickening & $79(18)$ & $44(10)$ & $325(72)$ \\
Proximal dilatation & $74(16)$ & $102(23)$ & $272(61)$ \\
Distal bronchial dilatation & $70(16)$ & $43(9)$ & $335(75)$ \\
Parenchymal disease & $63(14)$ & $50(11)$ & $335(75)$ \\
\hline
\end{tabular}

*Diagnostic interpretation by either two or all three radiologists.

Table 2 Correlation between computed tomography and bronchography at segmental level. Values are No (\% of total number reported as sharing each feature on bronchography)

\begin{tabular}{|c|c|c|c|c|c|c|}
\hline \multirow{3}{*}{$\begin{array}{l}\text { Computed } \\
\text { tomography }\end{array}$} & \multicolumn{6}{|c|}{ Bronchography } \\
\hline & \multicolumn{2}{|c|}{ Proximal dilatation } & \multicolumn{2}{|c|}{ Distal dilatation and non-tapering } & \multicolumn{2}{|c|}{ Bronchiectasis } \\
\hline & Present & Absent & Present & Absent & Present & Absent \\
\hline $\begin{array}{l}\text { Present } \\
\text { Absent }\end{array}$ & $\begin{array}{l}34(56) \\
27(44)\end{array}$ & $\begin{array}{r}40(10) \\
347(90)\end{array}$ & $\begin{array}{l}58(52) \\
54(48)\end{array}$ & $\begin{array}{c}21(6) \\
315(94)\end{array}$ & $\begin{array}{l}65(66) \\
33(34)\end{array}$ & $\begin{array}{c}27(8) \\
323(92)\end{array}$ \\
\hline
\end{tabular}

Present: feature definitely present (reported by two or three radiologists); absent: feature doubtful or absent (reported by only one or no radiologists).

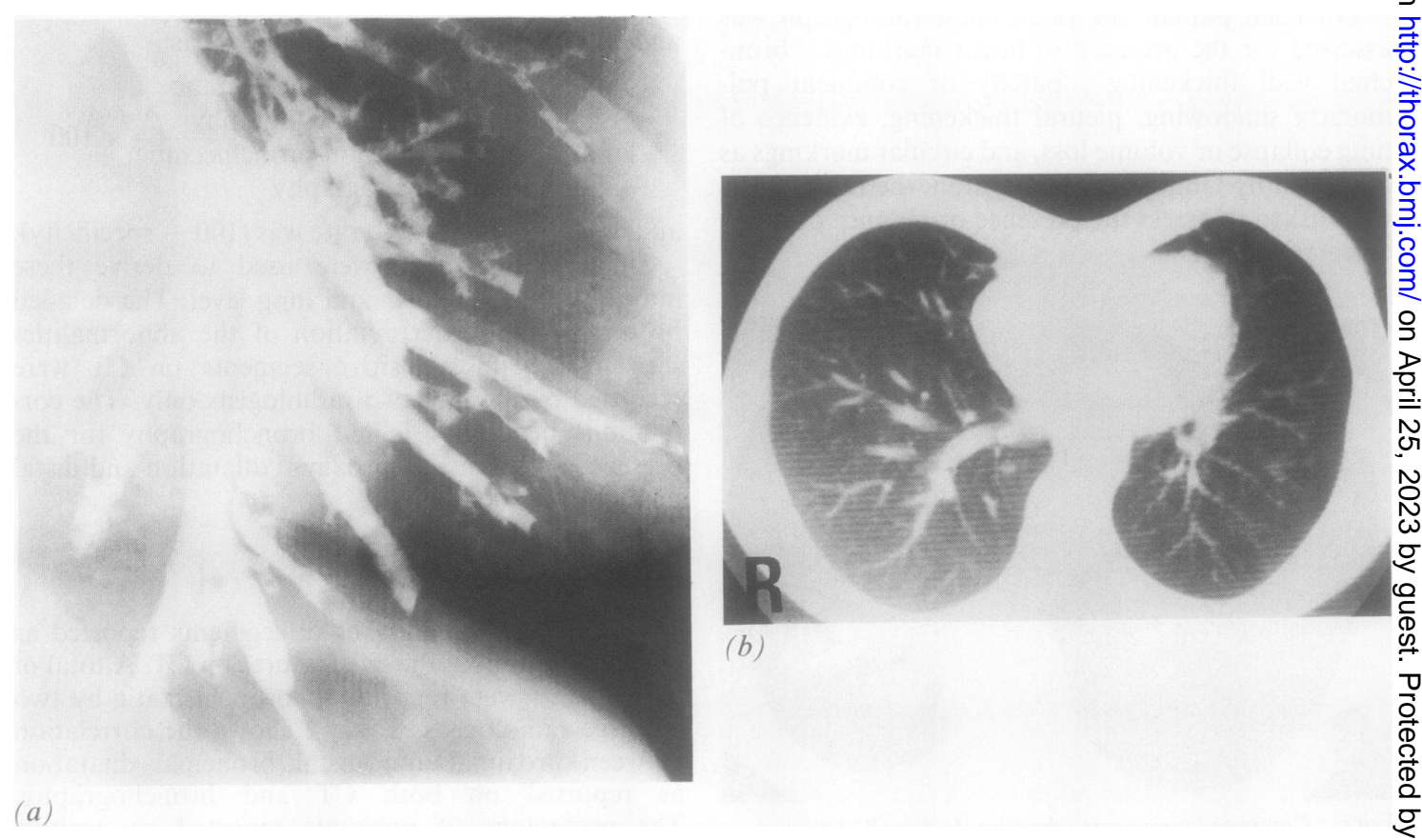

Fig 2 (a) Bronchogram of left lower lobe, showing cylindrical bronchiectasis. (b) Normal computed tomogram of both bases of the same patient. 

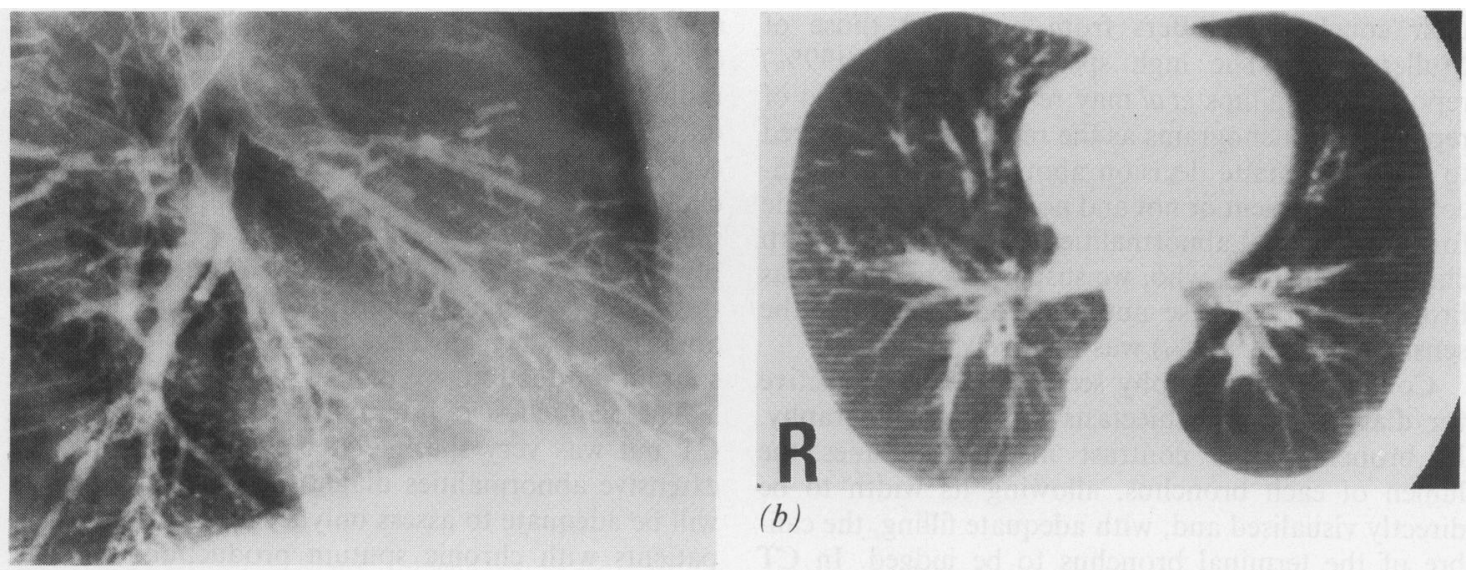

(a)

Fig 3 (a) Normal bronchogram of the left middle and lower lobes. (b) Computed tomogram of same patient, showing crowded, thick walled bronchi in the left lower lobe diagnosed as showing bronchiectasis.

these reported as positive for each feature on bronchography. The false positive rate for these features on CT was $10 \%$ and $6 \%$, respectively.

A total of 98 segments were reported as bronchiectatic on bronchography, but only 65 of these segments were reported as positive on CT. The sensitivity of CT in detecting bronchiectasis at a segmental level, therefore, was $66 \%(65 / 98)$, with a false negative rate of $34 \%$ (33/98) (fig $2 a$ and $b$ ). At segmental level the specificity of CT was $92 \%(323 / 350)$, with an $8 \%$ false positive rate $(27 / 350$ ) (fig $3 a$ and $3 b$ ). At lobar level the sensitivity was $71 \%(32 / 45)$ and specificity $86 \%(70 / 81)$, and at lung level the sensitivity was $87 \%(26 / 30)$ and specificity was $75 \%$ $(15 / 20)$.

Bronchiectasis was reported in 27 segments on CT alone with no confirmation on bronchography. The interpretation of these segments on the bronchograms by the first two radiologists was that nine were underfilled, eight showed chronic bronchitis, four were normal, and the other six segments were reported by one of the radiologists as having bronchiectasis (two) and an unspecified bronchial abnormality (four).

The sensitivity of the plain chest $x$ ray film for assessing bronchiectasis was only $37 \%(11 / 30)$ at lung level, with a $63 \%$ false negative rate compared with bronchography. The specificity was $95 \%(18 / 19)$, with a $5 \%$ false positive rate.

\section{Discussion}

The design of a trial to evaluate the sensitivity and specificity of CT is important. If patients are "positively" selected for CT by a bronchogram that shows bronchiectasis then a good correlation between the two investigations can be expected. A sufficient number of patients need to be examined to provide a representative sample of severity and types of bronchiectasis and other bronchial abnormalities. In our study 27 patients were studied consecutively, affording direct comparison of 50 lungs, which were separately assessed both with regard to CT and bronchography. Three pulmonary radiologists were asked to report on each investigation separately without knowledge of the results of the other investigation. If CT scan and bronchogram are performed close together and viewed simultaneously the diagnosis on CT may be influenced by the abnormalities seen on the bronchogram. The range of bronchographic abnormalities seen in our study ${ }^{6}$ varied from none (five of 50 lungs) to obvious bronchiectasis (19 lungs). Bronchographic features of both bronchiectasis and chronic bronchitis were present in 10 lungs. The bronchiectatic segments were varicose or cylindrical in all but one lung, which showed saccular (cystic) bronchiectasis. We contend that the range of abnormalities, the number of lungs studied, and the comparisons made were adequate to draw firm conclusions.

Our conclusions are similar to those of Muller et al, who analysed CT scan and bronchogram abnormalities at lobar level only but found poor correlation between the two investigations, with agreement in only six of 13 patients. ${ }^{4}$ In contrast, Mootoosamy et al found an excellent correlation between findings on CT and bronchography in their series, though there were 10 "false negative" segments on CT of 52 that showed bronchiectasis on bronchography (sensitivity $81 \%$ ) and four "false positive" segments on CT of 78 that did not show bronchiectasis on bronchography (specificity $95 \%$ ). ${ }^{3}$ The results of Mootoosamy et al are therefore similar to ours, though 
their conclusion ${ }^{3}$ differs from ours and those of Muller et al. ${ }^{4}$ The high specificity of CT $(99 \%)$ reported by Phillips et al may reflect their method of reporting bronchograms as the radiologist was asked to make a definite decision about whether bronchiectasis was present or not and no allowance was made for the bronchial abnormalities seen in patients with chronic bronchitis, who, we suspect, were labelled as bronchiectatic. ${ }^{7}$ These authors' concluded that the sensitivity of CT $(79 \%)$ was relatively poor.

Computed tomography seems to be less sensitive for diagnosing bronchiectasis than bronchography. In bronchography contrast medium outlines the lumen of each bronchus, allowing its width to be directly visualised and, with adequate filling, the calibre of the terminal bronchus to be judged. In CT assessment of bronchial dilatation depends on visualisation of the bronchial walls. With proximal bronchi, the walls are easily visible so that dilatation can be evaluated, though it is seen more easily in bronchi that run in the plane of the scan rather than in those that run axially. Distal bronchi with normal wall thickness cannot be seen with current computed tomographic scanners so that distal dilatation cannot be judged easily and bronchial walls need to be thickened to be visible.

The caution necessary in diagnosing bronchiectasis on CT, as it is a new technique, will also tend to reduce its sensitivity. There were a similar number of false positive and false negative diagnoses on CT, so that although a less conservative approach to the diagnosis of bronchiectasis on CT may improve the sensitivity, it would be offset by a reduction in specificity. A new technique for pulmonary CT has been described that incorporates a $3 \mathrm{~mm}$ slice width (rather than the conventional $8 \mathrm{~mm}$ or $10 \mathrm{~mm}$ ) with a different computer algorithm to produce sharper, clearer images. ${ }^{8}$ This "thin section" scan has not been evaluated critically with respect to lung diseases, but its usefulness in bronchiectasis has been implied.

The specificity of CT calculated at segmental level $(92 \%)$ is high and largely reflects the high frequency of normal segments on both CT and bronchography in the upper lobes (excluding the lingula). We consider the specificity at lung level $(75 \%)$ to be a more appropriate judge of CT.

The meaning of the abnormalities in the $27 \mathrm{seg}$ ments diagnosed as bronchiectatic on CT alone is unknown. Fourteen were regarded as abnormal on the bronchogram and eight were reported by two or three radiologists as showing features of chronic bronchitis. Nine other segments, all in one patient, were underfilled, leaving the possibility that bronchographic bronchiectasis was missed in some of these segments.

Although bronchography has been accepted as the established radiological method for diagnosing bronchiectasis, we have shown that two experienced radiologists found difficulty in some cases in differentiating between mild cylindrical bronchiectasis and chronic bronchitis. ${ }^{6}$ Clinical, bronchographic, and pathological similarities between these conditions are known to exist. ${ }^{69}$ There is possibly similar overlap of abnormal features on CT and this investigation may identify a different subgroup of abnormal bronchi present in patients with chronic sputum production.

The plain chest radiograph was less sensitive than CT but was very specific. A chest radiograph with extensive abnormalities diagnostic of bronchiectasis will be adequate to assess only a small proportion of patients with chronic sputum production. CT will identify more areas of bronchiectasis than a plain chest radiograph, but bronchography remains the definitive investigation for diagnosis of bronchiectasis in view of the poor sensitivity and significant false positive rate of CT. CT does have a place, however, in assessing patients with chronic sputum production who are unfit for, or refuse, bronchography and, if disease is identified, may aid in patient positioning during postural drainage and physiotherapy. It may be valuable, in combination with an initial bronchogram, in monitoring disease progression. If more accurate characterisation and localisation of disease is required for patients in whom surgery is considered CT alone is inadequate and bronchography should be performed. CT has the advantage of not being confined by historical terminology, which often restricts the radiologist to a differential diagnosis of chronic bronchitis or bronchiectasis on bronchography. As CT outlines different features from those seen on bronchography it may help us to understand the different types of disease and their natural histories in patients with sputum production, particularly if CT appearances are correlated with pathological features.

DC is supported by the Chest, Heart and Stroke Association.

\section{References}

1 Ansell G. Bronchography. In: Ansell G, ed. Complications in diagnostic radiology. Oxford: Alden Press, 1976:278-300.

2 Naidich DP, McCauley DI, Nagi F, Khouri NF, Stitik FP, Siegelman SS. Computed tomography of bronchiectasis. J Comput Assist Tomogr 1982;6:437-44.

3 Mootoosamy IM, Resnek RA, Rees RSO, Green M. Assessment of bronchiectasis by computed tomography. Thorax 1985;40:920-4.

4 Muller NL, Bergin CJ, Ostrow DN, Nichols DM. Role 
of computed tomography in the recognition of bronchiectasis. AJR 1984;143:971-6.

5 Güdbjerg CE. Roentgenologic diagnosis of bronchiectasis. Acta Radiol 1955;43:209-26.

6 Currie DC, Cooke J, Morgan AD, et al. Interpretation of bronchograms and chest radiographs in patients with chronic sputum production. Thorax 1987;42:278-84.

7 Phillips MS, Williams MP, Flower CDR. How useful is computed tomography in the diagnosis and assessment of bronchiectasis? Clin Radiol 1986;37:321-5.

8 Strickland B, Brennan J, Denison DM. Computed tomography in diffuse lung disease: improving the image. Clin Radiol 1985;37:335-8.

9 Reid L. Measurement of the bronchial mucous gland layer: a diagnostic yardstick in chronic bronchitis. Thorax 1960;15:132-41.

\section{Book notices}

International Trends in General Thoracic Surgery - Volume I: Lung Cancer. NC Delarue, H Eschapasse. (Pp 315; £47.25 hardback.) Philadelphia: WB Saunders, 1985. ISBN 0-7216-1349-7.

The scope of this initial volume in a new series on general thoracic surgery is indeed international and broad. Although certain chapters are purely technical (the procedures of choice for tumours of the chest wall and the main carina and the occasional role of surgery in superior vena caval obstruction), there is much of general respiratory interest. The volume begins with histogenesis and moves through considerations of the merits of staging lung cancer, mediastinoscopy, preoperative assessment and the management of occult tumours. Although the list of contributors is distinguished some of the contributions merely summarise the data from the particular unit and much of the presentation is personalised, which limits its value slightly in the context of the problem as a whole. This is balanced in some areas of controversy by a few pages of critical discussion from another centre. The volume covers the whole of surgery thoroughly for non-small cell lung cancer and then includes sections on small cell cancer, discussing chemotherapy alone, preoperative and postoperative chemotherapy, interferon treatment, and the role of monoclonal antibodies. The book is unusual in that the contributions are based on individuals units' experience and therefore many controversial statements go unchallenged. It is, however, presented with up to date, very adequate references. All in all the problems that beset those trying to improve the outcome for patients with lung cancer are clearly outlined.
Asthma: Clinical, Pharmacology, and Therapeutic Progress. AB Kay. (Pp 412; figs; $£ 42$ hardback.) Oxford: Blackwell Scientific Publications, 1986. ISBN 0-632-01465-2.

This book consists of a compilation of the lectures given in November 1984 at the annual course on the pharmacology of asthma at the Cardiothoracic Institute, London. It consists of 33 chapters, from 60 international contributors, designed to give a "state of the art" update on mechanisms of asthma and the mode of action of the drugs most commonly used in its treatment. The emphasis through much of the volume is on areas of "current" research interest and several chapters are basically preliminary disclosures of research data, much of which has subsequently been published in full. What would undoubtedly have been a stimulating strength of the course in 1984 tends to detract from the book 18 months later, and these elements might more purposefully have appeared more rapidly as a proceedings supplement of a respiratory journal. In other ways too the balance of interest, though reasonable in 1984, now seems dated-for example, three chapters and 32 pages on leukotrienes and only one chapter and exactly a third of the pages for prostaglandins, with little mention of recent interest in the latter's possible role in the late asthmatic reaction and induction of airway hyperresponsiveness, or in the role of $\mathrm{PGE}_{2}$ in control of airways smooth muscle. Nevertheless, despite these criticisms the book succeeds well it its general strategy, and even in the areas in which knowledge is advancing rapidly-for example, asthma as an inflammatory airway disease, the possible and presumptive mechanisms are clearly described. In the more established areas of mast cell and smooth muscle physiology, pharmacology, and therapeutics the volume includes excellent review chapters. The book is easy to read and generally very well illustrated and has numerous references. It should succeed in its aim of appealing both to the clinician and, at least as introductory reading, to the research scientist.-EHW 